\title{
First-Passage Time Moment Approximation for the General Diffusion Process to a General Moving Barrier
}

\author{
Basel Mohammad Said Al-Eideh \\ Department of Quantitative Methods and Information System, College of Business Administration, Kuwait University, Showaikh, Kuwait \\ Email address: \\ basel@cba.edu.kw \\ To cite this article: \\ Basel Mohammad Said Al-Eideh. First-Passage Time Moment Approximation for the General Diffusion Process to a General Moving \\ Barrier. American Journal of Theoretical and Applied Statistics. Vol. 7, No. 5, 2018, pp. 167-172. doi: 10.11648/j.ajtas.20180705.11
}

Received: June 13, 2018; Accepted: July 9, 2018; Published: August 2, 2018

\begin{abstract}
The problem of determining the first-passage times to a moving barrier for diffusion and other Markov processes arises in biological modeling, population growth, statistics, engineering, etc. Since the development of mathematical models for population growth of great importance in many fields. Therefore, the growth and decline of real populations can, in many cases, be well approximated by the solutions of stochastic differential equations. However, there are many solutions in which the essentially random nature of population growth should be taken into account. This paper focusses in approximating the moments of the first - passage time for the general diffusion process to a general moving barrier. This was done by approximating the differential equations by equivalent difference equations.
\end{abstract}

Keywords: First Passage Time, General Diffusion Process, Difference Equations, General Moving Barrier

\section{Introduction}

A first passage time in a stochastic system, is the time taken for a state variable to reach a certain value. The idea that a first passage time of a stochastic process might describe the time to occurrence of an event has a long history, starting with an interest in the first passage time of Wiener diffusion processes in economics and then in physics in the early 1900s. Modeling the probability of financial ruin as a first passage time was an early application in the field of insurance. Historically, an interest in the mathematical properties of first-passage times and statistical models and methods for analysis of survival data appeared steadily between the middle and end of the 20th century.

Therefore, first - passage time play an important role in the area of applied probability theory especially in stochastic modeling. Several examples of such problems are the extinction time of a branching process, or the cycle lengths of a certain vehicle actuated traffic signals. Actually the first passage times to a moving barriers for diffusion and other markov processes arises in biological modeling (Cf. Ewens [1]), in statistics (Cf. Darling and Siegert [2] and Durbin [3]).

Different authors have been studied many important results related to the first - passage time from different points of view. For example, McNeil [4] has derived the distribution of the integral functional, where is the first - passage time to the origin in a general birth - death process with $X(0)=x$ and $g($.$) is an arbitrary function. Also, a number of classical$ birth and death processes upon taking diffusion limits to asymptotically approach the Ornstein - Uhlenbeck (O. U.) have been shown by Iglehart [5], McNeil and Schach [6].

Moreover, many properties such as the first - passage time to a barrier, absorbing or reflecting, located some distance from an initial starting point of the $\mathrm{O}$. U. process and the related diffusion process such as the case of the first passage time of a Wiener process to a linear barrier is a closed form expression for the density available is discussed in Cox and Miller [7]. Also, others such as, Karlin and Taylor [8], Thomas [9], Ferebee [10], Tuckwell and Wan [11], Alawneh and A-Eideh [12], Al-Eideh [13-15], etc. have been discussed the first passage time from different points of view.

In particular, Thomas [9] describes some mean first passage time approximation for the Ornstein - Uhlenbeck process. Tuckwell and Wan [11] have studied the firstpassage time of a Markov process to a moving barrier as a first-exit time for a vector whose components include the process and the barrier.

Alawneh and A-Eideh [12], describes some mean firstpassage time approximation for the Ornstien-Uhlenbeck process with a single absorbing barrier using the method of 
approximating the differential equations by difference equations.

Al-Eideh [13], has discussed the problem of finding the moments of the first passage time distribution for the birthdeath diffusion to a moving linear barrier using the same method of approximating the differential equations by difference equations. Furthermore, in [15], he considered the case of birth-death diffusion with immigrations to a moving linear barrier.

In addition, Al-Eideh [14], has discussed the problem of finding the moments of the first passage time distribution for the Wright-Fisher diffusion processes to a single absorbing barrier using the method of approximating the differential equations by difference equations, too.

In this paper, we consider the general diffusion process and study the first - passage time for such a process to a general moving barrier. More specifically, the moment approximations are derived using the method of difference equations. Note that the same lines as in Al-Eideh [15] will be followed but the derivations, the generalizations are not straightforward, and the results are very different.

\section{Moment Approximations for the First - Passage Time}

This section will be devoted to derive the moment approximation for the first passage time for a general diffusion process to a general linear barrier.

Suppose that $\left\{X_{t}: t \geq 0\right\}$ be the general diffusion Process with infinitesimal mean $\mu(x)$ and variance $\sigma^{2}(x)$ starting at some $x_{0}>0$. Also, $\left\{X_{t}: t \geq 0\right\}$ is a Markov process with state space $S=[0, \infty)$ and satisfies the Ito stochastic differential equation

$$
d X_{t}=\mu\left(X_{t}\right) d t+\sigma\left(X_{t}\right) d W_{t}
$$

Here $\left\{W_{t}: t \geq 0\right\}$ is a standard Wiener process with mean zero and variance $t$. The existence and uniqueness conditions are assumed to be satisfied (Cf. Gikman and Skorohod [16]). Let $\{Y(t): t \geq 0\}$ be a general moving barrier equation such that $Y(t)=h(t)$, with $Y(0)=h(0)$. Or equivalently

$$
\frac{d Y(t)}{d t}=h^{\prime}(t)
$$

Assume the random variables $T_{y}$ denotes the first passage time of a process $X_{t}$ to a general moving barrier $Y(t)=h(t)$ such that

$$
\mathrm{T}_{\mathrm{Y}}=\inf \left\{\mathrm{t} \geq 0: \mathrm{X}_{\mathrm{t}} \geq \mathrm{h}(\mathrm{t})\right\}
$$

Now, letting $\mathrm{p}\left(x_{0}, \mathrm{x} ; \mathrm{t}\right)$ is the probability density function of $\mathrm{X}_{\mathrm{t}}$ conditional on $\mathrm{X}_{\mathrm{t}}=x_{0}$, then the probability density function of $T_{y}$ can be written as

$$
g\left(t ; \quad x_{0}\right)=-\frac{d}{d t} \int_{-\infty}^{h(t)} p\left(x_{0}, x ; t\right) d x
$$

Suppose that the $n$-th moment of the first - passage time $\mathrm{T}_{\mathrm{Y}}$ be $\mathrm{M}_{\mathrm{n}}\left(\mathrm{x}_{0}, \mathrm{Y} ; \mathrm{t}\right) ; \mathrm{n}=1,2,3, \ldots \ldots$, , then

$$
M_{n}\left(x_{0}, Y ; t\right)=E\left(T_{Y}^{n}\right) ; n=1,2,3, \ldots,
$$

Using the forward Kolmogorov equation, it follows that the n-th moment of $T_{Y}$ must satisfy the ordinary differential equation

$$
\begin{aligned}
& \sigma(\mathrm{x}) \mathrm{M}_{\mathrm{n}}^{\prime \prime}\left(\mathrm{x}_{0}, \mathrm{Y} ; \mathrm{t}\right)+\mu(\mathrm{x}) \mathrm{M}_{\mathrm{n}}^{\prime}\left(\mathrm{x}_{0}, \mathrm{Y} ; \mathrm{t}\right) \\
& +h^{\prime}(t) M_{n}^{\prime}\left(x_{0}, Y ; t\right)=-n M_{n-1}\left(x_{0}, Y ; t\right)
\end{aligned}
$$

Or equivalently

$$
\begin{aligned}
& M_{n}^{\prime \prime}\left(x_{0}, Y ; t\right)+\frac{\mu(x)}{\sigma(x)} M_{n}^{\prime}\left(x_{0}, Y ; t\right) \\
& +\frac{h^{\prime}(t)}{\sigma(x)} M_{n}^{\prime}\left(x_{0}, Y ; t\right) \\
& =-\frac{n}{\sigma(x)} M_{n-1}\left(x_{0}, Y ; t\right)
\end{aligned}
$$

Note that here $\mathrm{M}_{\mathrm{n}}^{\prime}\left(\mathrm{x}_{0}, \mathrm{Y} ; \mathrm{t}\right)$ and $\mathrm{M}_{\mathrm{n}}^{\prime \prime}\left(\mathrm{x}_{0}, \mathrm{Y} ; \mathrm{t}\right)$ are the first and the second derivatives of $\mathrm{M}_{\mathrm{n}}\left(\mathrm{x}_{0}, \mathrm{Y}\right.$; $\left.\mathrm{t}\right)$ with respect to $x$ and appropriate boundary conditions for $\mathrm{n}=1,2,3, \ldots$ besides $\mathrm{M}_{0}\left(\mathrm{x}_{0}, \mathrm{Y} ; \mathrm{t}\right)=1$ where $\mathrm{x}_{0} \leq \mathrm{x} \leq \mathrm{Y}$.

Rewriting equation (5), we get

$$
\begin{aligned}
& M_{n}^{\prime \prime}\left(x_{0}, Y ; t\right)=-\frac{n}{\sigma(x)} M_{n-1}\left(x_{0}, Y ; t\right) \\
& -\left(\frac{\mu(x)}{\sigma(x)}+\frac{h^{\prime}(t)}{\sigma(x)}\right) M_{n}^{\prime}\left(x_{0}, Y ; t\right)
\end{aligned}
$$

By letting $\Delta$ to be the difference operator, then the first order difference equation of $M_{n}\left(x_{0}, Y ; t\right)$ will be defined as (Cf. Kelley and Peterson [17]):

$$
\Delta M_{n}\left(x_{0}, Y ; t\right)=M_{n+1}\left(x_{0}, Y ; t\right)-M_{n}\left(x_{0}, Y ; t\right)
$$

Now approximating the equation in (6), we find that

$$
\begin{aligned}
& M_{n}^{\prime \prime}\left(x_{0}, Y ; t\right)=-\frac{n}{\sigma(x)} M_{n-1}\left(x_{0}, Y ; t\right) \\
& -\left(\frac{\mu(x)}{\sigma(x)}+\frac{h^{\prime}(t)}{\sigma(x)}\right) \Delta M_{n}\left(x_{0}, Y ; t\right)
\end{aligned}
$$

Using equation (7), then equation (8) can be rewritten as follows: 


$$
\begin{aligned}
M_{n}^{\prime \prime}\left(x_{0} Y ; t\right) & =-\frac{n}{\sigma(x)} M_{n-1}\left(x_{0}, Y ; t\right) \\
& +\left(\frac{\mu(x)}{\sigma(x)}+\frac{h^{\prime}(t)}{\sigma(x)}\right) M_{n}\left(x_{0}, Y ; t\right) \\
& -\left(\frac{\mu(x)}{\sigma(x)}+\frac{h^{\prime}(t)}{\sigma(x)}\right) M_{n+1}\left(x_{0}, Y ; t\right)
\end{aligned}
$$

Now, by letting

$$
A=\left[\begin{array}{cc}
\left(\frac{\mu(\mathrm{x})}{\sigma(\mathrm{x})}+\frac{h^{\prime}(\mathrm{t})}{\sigma(\mathrm{x})}\right) & -\left(\frac{\mu(\mathrm{x})}{\sigma(\mathrm{x})}+\frac{h^{\prime}(\mathrm{t})}{\sigma(\mathrm{x})}\right) \\
-\frac{2}{\sigma(\mathrm{x})} & \left(\frac{\mu(\mathrm{x})}{\sigma(\mathrm{x})}+\frac{h^{\prime}(\mathrm{t})}{\sigma(\mathrm{x})}\right) \\
0 & -\frac{3}{\sigma(\mathrm{x})} \\
0 & 0 \\
\vdots & \vdots
\end{array}\right.
$$

Also, letting

$$
\frac{d \vec{M}\left(x_{0}, Y ; t\right)}{d x}=\vec{R}\left(x_{0}, Y ; t\right)
$$

Consequently, this imply

$$
\frac{d^{2} \vec{M}\left(x_{0}, Y ; t\right)}{d x^{2}}=\frac{d \vec{R}\left(x_{0}, Y ; t\right)}{d x}
$$

Now, by applying to equation (10), we obtain

$$
\frac{d}{d x}\left[\begin{array}{l}
\vec{R}\left(x_{0}, Y ; t\right) \\
\vec{M}\left(x_{0}, Y ; t\right)
\end{array}\right]=\left[\begin{array}{cc}
0 & A \\
I & 0
\end{array}\right] \cdot\left[\begin{array}{l}
\vec{R}\left(x_{0}, Y ; t\right) \\
\vec{M}\left(x_{1}, t_{0}\right)
\end{array}\right]
$$

$$
\vec{M}\left(x_{0}, Y ; t\right)=\left[M_{1}\left(x_{0}, Y ; t\right), M_{2}\left(x_{0}, Y ; t\right), \cdots\right]
$$

and using the matrix theory to solve the differential equation defined in equation (9), we get

$$
\frac{d^{2} \vec{M}\left(x_{0}, Y ; t\right)}{d x^{2}}=A \vec{M}\left(x_{0}, Y ; t\right)
$$

Where

$$
\left.\begin{array}{ccc}
0 & 0 & \cdots \\
-\left(\frac{\mu(x)}{\sigma(x)}+\frac{h^{\prime}(t)}{\sigma(x)}\right) & 0 & \cdots \\
\left(\frac{\mu(x)}{\sigma(x)}+\frac{h^{\prime}(t)}{\sigma(x)}\right) & -\left(\frac{\mu(x)}{\sigma(x)}+\frac{h^{\prime}(t)}{\sigma(x)}\right) & \cdots \\
-\frac{4}{\sigma(x)} & \left(\frac{\mu(x)}{\sigma(x)}+\frac{h^{\prime}(t)}{\sigma(x)}\right) & \cdots \\
\vdots & \ddots & \ddots
\end{array}\right]
$$

then given explicitly by:

$$
\left[\begin{array}{l}
\vec{R}\left(\mathrm{x}_{0}, \mathrm{Y} ; \mathrm{t}\right) \\
\overrightarrow{\mathrm{M}}\left(\mathrm{x}_{0}, \mathrm{Y} ; \mathrm{t}\right)
\end{array}\right]=\mathrm{e}^{\left[\begin{array}{ll}
0 & \mathrm{~A}^{*} \\
\mathrm{D} & 0
\end{array}\right]} \cdot\left[\begin{array}{l}
\overrightarrow{\mathrm{R}}\left(\mathrm{x}_{0}, \mathrm{Y} ; \mathrm{t}\right) \\
\vec{M}\left(\mathrm{x}_{\mathrm{r}} \mathrm{Y} ; \mathrm{t}_{0}\right)
\end{array}\right]
$$

Where $D=\left[d_{i j}\right] ; i, j \geq 1$ is the diagonal matrix with entries

$$
d_{i j}=\left\{\begin{array}{cc}
\left(h(t)-x_{0}\right) & ; j=i \\
0 & ; \text { Otherwise }
\end{array}\right.
$$

And $A^{*}=\left[a_{i j}^{*}\right] ; i, j \geq 1$ is the matrix with entries

Where 0 is the zero matrix and $I$ is the identity matrix.

Therefore, the solution of the system of equation in (13) is

$$
a_{i j}^{*}=\left\{\begin{array}{c}
-\frac{i}{\sigma(x)} \ln \left(\frac{h(t)}{x_{0}}\right) \quad ; j=i-1 \\
\left(\frac{\mu(x)}{\sigma(x)}+\frac{h^{\prime}(t)}{\sigma(x)}\right)\left(h(t)-x_{0}\right) \quad ; j=i \\
-\left(\frac{\mu(x)}{\sigma(x)}+\frac{h^{\prime}(t)}{\sigma(x)}\right)\left(h(t)-x_{0}\right) \quad ; j=i+1 \\
0 \quad ; \text { Otherwise }
\end{array}\right.
$$

Note that by Taylor expansion, the matrix $e^{B}$ where $B=\left[\begin{array}{ll}0 & A^{*} \\ D & 0\end{array}\right]$ can be defined as

$$
\mathrm{e}^{\mathrm{B}}=\mathrm{I}+\mathrm{B}+\frac{\mathrm{B}^{2}}{2 !}+\frac{\mathrm{B}^{3}}{3 !}+\ldots \ldots \ldots \ldots
$$

Moreover, according to Zeifman [18] and since it is a Cauchy operator of equation (2.6), this series is convergent.

\section{Applications}

In this section, we will introduce some applications of finding the first passage time moments approximations for some diffusion processes to a linear moving barrier, where 
$Y(t)=c t+k$, with $Y(0)=k$. Or equivalently

$$
\frac{d Y(t)}{d t}=c
$$

Note that the first - passage time of a process $X_{t}$ in (2) becomes $\mathrm{T}_{\mathrm{Y}}=\inf \left\{\mathrm{t} \geq 0: \mathrm{X}_{\mathrm{t}} \geq \mathrm{ct}+\mathrm{k}\right\}$.

\subsection{The Write-Fisher Diffusion Model for Gene Frequency}

Consider the simplest Write-Fisher diffusion model for depicting fluctuations of gene frequency of the A-type within a population having both A-and a-types subject to selection influences with the state space $[0,1]$ and diffusion coefficients

$$
\mu(x)=\sigma x(1-x) \text { and } \sigma^{2}(x)=x(1-x)
$$

Thus, the first - passage time moments is given explicitly by solving (14) with $D=\left[d_{i j}\right] ; i, j \geq 1$ is the diagonal matrix with entries

$$
d_{i j}=\left\{\begin{array}{cc}
\left(c t+k-x_{0}\right) \quad ; j=i \\
0 \quad ; \text { Otherwise }
\end{array}\right.
$$

And $A^{*}=\left\lfloor a_{i j}^{*}\right\rfloor ; i, j \geq 1$ is the matrix with entries

$$
a_{i j}^{*}=\left\{\begin{array}{c}
-\frac{i}{\sqrt{x(1-x)}} \ln \left(\frac{c t+k}{x_{0}}\right) \quad ; j=i-1 \\
\left(\sigma+\frac{c}{\sqrt{x(1-x)}}\right)\left(c t+k-x_{0}\right) \quad ; j=i \\
-\left(\sigma+\frac{c}{\sqrt{x(1-x)}}\right)\left(c t+k-x_{0}\right) \quad ; j=i+1 \\
0 \quad ; \text { Otherwise }
\end{array}\right.
$$

\subsection{The Bessel Process}

The Bessel process $\{X(t)\}$ of parameter $\alpha \geq 0$ is the one-dimensional diffusion process on $[0, \infty)$ having the infinitesimal coefficients

$$
\mu(x)=\frac{\alpha-1}{2 x} \text { and } \sigma^{2}(x)=1
$$

With explicit transition density given by

$$
p(t, x, y)=\frac{\exp \left[-\left(x^{2}+y^{2}\right) / 2 t\right]}{t(x y)^{(\alpha-2) / 2}} y^{\alpha-1} I_{(\alpha-2) / 2}\left(\frac{x y}{t}\right) ; t>0, x, y>0
$$

Where $I_{v}(z)$ is the modified Bessel function

$$
I_{v}(z)=\sum_{k=0}^{\infty} \frac{(z / 2)^{2 k+v}}{k ! \Gamma(k+v+1)}
$$

Thus, the first - passage time moments is given explicitly by solving (14) with $D=\left[d_{i j}\right] ; i, j \geq 1$ is the diagonal matrix with entries

$$
d_{i j}=\left\{\begin{array}{cc}
\left(c t+k-x_{0}\right) \quad ; j=i \\
0 \quad ; \text { Otherwise }
\end{array}\right.
$$

And $A^{*}=\left[a_{i j}^{*}\right] ; i, j \geq 1$ is the matrix with entries 


$$
a_{i j}^{*}=\left\{\begin{array}{c}
-i \ln \left(\frac{c t+k}{x_{0}}\right) \quad ; j=i-1 \\
\left(\frac{\alpha-1}{2 x}+c\right)\left(c t+k-x_{0}\right) \quad ; j=i \\
-\left(\frac{\alpha-1}{2 x}+c\right)\left(c t+k-x_{0}\right) \quad ; j=i+1 \\
0 \quad ; 0 \text { therwise }
\end{array}\right.
$$

\subsection{The Jacobi Diffusion Process}

The Jacobi diffusion process $\{\mathrm{X}(\mathrm{t})\}$ of positive parameter $\alpha$ and $\beta$ is the one-dimensional diffusion process on $I=(-1,1)$ where the boundaries -1 and 1 are the entrance boundaries and having the infinitesimal coefficients

$$
\mu(\mathrm{x})=\frac{1}{2}[(\beta+1)(1-\mathrm{x})-(\alpha+1)(1+\mathrm{x})]
$$

and

$$
\sigma^{2}(\mathrm{x})=\left(1-\mathrm{x}^{2}\right)
$$

Thus, the first - passage time moments is given explicitly by solving (14) with $D=\left[\mathrm{d}_{\mathrm{ij}}\right] ; i, j \geq 1$ is the diagonal matrix with entries

$$
d_{i j}=\left\{\begin{array}{c}
\left(c t+k-x_{0}\right) \quad ; j=i \\
0 \quad ; \text { Otherwise }
\end{array}\right.
$$

And $A^{*}=\left\lfloor a_{i j}^{*}\right\rfloor ; i, j \geq 1$ is the matrix with entries

$$
a_{i j}^{*}=\left\{\begin{array}{l}
-\frac{i}{\sqrt{\left(1-x^{2}\right)}} \ln \left(\frac{c t+k}{x_{0}}\right) \quad ; j=i-1 \\
\left(\frac{\frac{1}{2}[(\beta+1)(1-x)-(\alpha+1)(1+x)]+c}{\sqrt{\left(1-x^{2}\right)}}\right)\left(c t+k-x_{0}\right) \\
-\left(\frac{\frac{1}{2}[(\beta+1)(1-x)-(\alpha+1)(1+x)]+c}{\sqrt{\left(1-x^{2}\right)}}\right)\left(c t+k-x_{0}\right) \\
0
\end{array}\right.
$$

\section{Conclusion}

In conclusion, the system of the solutions in equation (14) gives an explicit solution to the first - passage time moments for the general diffusion process to a general moving barrier. Therefore, this technique is very beneficial in using the difference equation to approximate the ordinary differential equation since it is the discretization of the ODE. In addition, this general explicit solution can capture all cases of diffusion processes with known infinitesimal coefficients to any functional form of moving barrier. In general, this increases the applicability of diffusion processes in all area of applied probability theory, especially, in stochastic modeling.

\section{References}

[1] W. J. Ewens, Mathematical Population Genetics. SpringerVerlag, Berlin (1979).

[2] D. Darling and A. J. F. Siegert, The first - passage problem for a continuous Markov process. Ann. Math. Statist. 24 (1953), 624-639.

[3] J. Durbin, Boundary-crossing probabilities for the Brownian motion and Poisson processes and techniques for computing the power of the Kolmogorov-Smirnov test. J. Appl. Prob. 8 (1971), 431-453.

[4] D. R. McNeil, Integral functional of birth and death processes and related limiting distributions. Ann. Math. Statist. 41 (1970), 480-485. 
[5] D. L. Iglehart, Limiting diffusion approximation for the many server queue and the repairman problem. J. Appl. Prob. 2 (1965), 429-441.

[6] D. R. McNeil and S. Schach, Central limit analogues for Markov population processes. J. R. Statist. Soc. B, 35 (1973), $1-23$.

[7] D. R. Cox and H. D. Miller, The theory of stochastic processes. Methuen, London (1965).

[8] S. Karlin and H. M. Taylor, A Second Course in Stochastic Processes. Academic press. New York (1981).

[9] M. U. Thomas, Some mean first passage time approximations for the Ornstein - Uhlenbeck process. J. Appl. Prob. 12 (1975), 600-604.

[10] B. Ferebee, The tangent approximation to one-sided Brownian exit densities. Z. Wahrscheinlichkeitsth 61 (1982), 309-326.

[11] H. C. Tuchwell and F. Y. M. Wan, First-passage time of Markov processes to moving barriers. J. Appl. Prob. Vol. 21 (1984), 695-709.

[12] A. J. Alawneh and B. M. Al-Eideh, Moment approximation of the first-passage time for the Ornstien- Uhlenbeck process. Intern. Math. J, Vol. 1 (2002), No. 3, 255-258.
[13] B. M. Al-Eideh, First-passage time moment approximation for the birth-death diffusion process to a moving linear barrier. $J$. Stat. \& Manag. Systems, Vol. 7 (2004), No. 1, 173-181.

[14] B. M. Al-Eideh, First-passage time moment approximation for the write-fisher diffusion process with absorbing barrier. Intern. J. Contep. Math, Sciences, Vol. 5 (2010), No. 27, 1303-1308.

[15] B. M. AL-EIDEH, The Moment Approximation of the FirstPassage Time for the Birth-Death Diffusion Process with Immigrations to a Moving Linear Barrier. In Recent Advances in Automation Control, Modeling and Simulation. Edited by Fujita, H., Tuba, M., and Sasaki, J. WSEAS Press, April, (2013), 197-201.

[16] I. Gikman and A. V. Skorohod, The theory of stochastic processes. Springer-Verlag, Berlin and New York, (1974).

[17] W. G. Kelly and A. C. Peterson, Difference Equations: An Introduction with Applications. Academic Press, New York (1991).

[18] A. I. Zeifman, Some estimates of the rate of convergence for birth and death processes. J. Appl. Prob. 28 (1991), 268-277. 